\title{
The local discontinuous Galerkin method with generalized alternating flux for solving Burger's equation
}

\author{
Rongpei Zhang ${ }^{\mathrm{a}, *}$, Di Wang ${ }^{\mathrm{a}}$, Xijun Yu${ }^{\mathrm{b}}$, Bo Chen ${ }^{\mathrm{c}}$, Zhen Wang ${ }^{\mathrm{d}, *}$ \\ a School of Mathematics and Systematic Sciences, Shenyang Normal University, Shenyang 110034, P. R. China. \\ ${ }^{b}$ Laboratory of Computational Physics, Institute of Applied Physics and Computational Mathematics, Beijing 100088, P. R. China. \\ ${ }^{c}$ College of Mathematics and Statistics, Shenzhen University, Shenzhen 518060, P. R. China. \\ ${ }^{d}$ College of Mathematics and Systems Science, Shandong University of Science and Technology, Qingdao 266590, P. R. China.
}

\begin{abstract}
In this paper, we propose the local discontinuous Galerkin method based on the generalized alternating numerical flux for solving the one-dimensional nonlinear Burger's equation with Dirichlet boundary conditions. Based on the Hopf-Cole transformation, the original equation is transformed into a linear heat conduction equation with homogeneous Neumann boundary conditions. We will show that this method preserves stability. By virtue of the generalized Gauss-Radau projection, we can obtain the sub-optimal rate of convergence in $L^{2}$-norm of $\mathcal{O}\left(h^{k+\frac{1}{2}}\right)$ with polynomial of degree $k$ and grid size $h$. Numerical experiments are given to verify the theoretical results.
\end{abstract}

Keywords: Burger's equation, local discontinuous Galerkin method, Hopf-Cole transformation, generalized alternating numerical flux, generalized Gauss-Radau projection.

2010 MSC: 65M60.

(C)2019 All rights reserved.

\section{Introduction}

In this paper, we consider the following one-dimensional Burger's equation:

$$
u_{t}+u u_{x}-v u_{x x}=0, \quad a<x<b, \quad 0<t \leqslant T,
$$

with the initial condition

$$
u(x, 0)=f(x), \quad a<x<b,
$$

and the boundary conditions

$$
u(a, t)=0, \quad u(b, t)=0, \quad 0<t \leqslant T,
$$

where $v$ is a constant.

\footnotetext{
*Corresponding author

Email addresses: rongpeizhang@163.com (Rongpei Zhang), wangdisynu@gmail.com (Di Wang), yuxj@iapcm.ac.cn (Xijun Yu), chenbo@szu.edu.cn (Bo Chen), wangzhen.sd@gmail.com (Zhen Wang)
}

doi: $10.22436 /$ jnsa.012.05.04

Received: 2018-01-15 Revised: 2018-08-04 Accepted: 2018-10-06 
Burger's equation was first introduced by Bateman and was later referred to as Burger's equation after Burger introduced this equation as a mathematical model for fluid flow. The equation (1.1) has a wide range of applications such as gas dynamics, boundary layer behavior, turbulence, wave propagation, etc.. In the study of fluid dynamics, it can be considered as a simplified model of the Navier-Stokes equation by neglecting the pressure term. Simulation of Burger's equation is a natural first step towards developing methods for the computation of complex flows. Therefore, it is also considered as a test problem for validating several numerical algorithms.

For the analytical solution of the Burger's equation, Hopf and Cole respectively suggested that for any initial conditions the solution can be expressed in the form of Fourier series [12,14]. However, there are some difficulties to obtain the exact solution when the initial value is not smooth enough. Many researchers have developed various numerical methods for the solution of Burger's equation. The available numerical techniques include finite element method [7, 22], Local Crank-Nicolson method [15], the finite difference methods [4, 19], lattice Boltzmann method [13], Chebyshev spectral collocation method [18], cubic B-spline differential quadrature method [1, 2], boundary element method [5] and differential quadrature method [17]. More recently, Mukundan and Awasthi [21] developed the finite difference method coupled with an low-dispersion and low-dissipation implicit Runge-Kutta method for (1.1); Tamsir et al. proposed an algorithm based on exponential modified cubic B-spline differential quadrature method [25]; Jiwari developed the hybrid numerical scheme based on Euler implicit method, quasilinearization and uniform Haar wavelets [16]. Besides various numerical methods such as biorthogonal multiwavelets [3], RBF collocation methods [26], high order splitting methods [23] were also developed.

In this paper we study the local discontinuous Galerkin (LDG) method for solving equation (1.1). We first apply the Hopf-Cole transformation to transform the Burger's equation into a heat equation. Then the heat equation is rewritten into an equivalent system containing only first-order derivatives. The system is after discretized by the discontinuous Galerkin (DG) method to get the solution of the introduced variable. Finally we can get the numerical solution of the original equations by transforming back. The LDG method was first introduced by Cockburn and Shu [11] which was motivated by Bassi and Rebay's work on Navier-Stokes problems [6]. The LDG method possesses certain flexibility and advantage, such as, it is local (element-wise) conservative. It can also be easily designed for any order of accuracy. In fact, the order of accuracy can be locally determined in each cell. It is also suitable for complex domain and adaptive computation and allows a very efficient parallelization. These properties make it popular for practical computations.

The DG methods' popularity has also attracted researchers to solve the Burger's equations. Shao et al. have presented LDG method for a nonlinear Burger's equation with Dirichlet boundary conditions [24]. They have proved that the LDG method has the $(k+1)$-th order of convergence rate, while the numerical experiments show only $\left(k+\frac{1}{2}\right)$-th order convergence which is inconsistent with the theoretical results. In $[27,28]$, we have applied the direct DG method to Burger's, modified Burger's and coupled Burger's equation by LDG method. In the design of the LDG method, the key ingredient is the choice of numerical flux to ensure the stability and high order accuracy. The LDG methods above used the purely alternating numerical flux. The idea of this paper is motivated by work of Meng et al. [20], in which the DG methods is applied to solve linear hyperbolic problems based on upwind-biased numerical flux. Recently, Cheng et al. $[9,10]$ developed the LDG method with generalized numerical flux for convection-diffusion equations. They obtained the optimal error estimate based on the construction and analysis of the newly designed generalized Gauss-Radau projections. In this paper we will apply LDG method based on the generalized alternating numerical flux. By virtue of the generalized Gauss-Radau projection, we can prove that the proposed method can get sub-optimal rate of convergence in $L^{2}$-norm of $\mathcal{O}\left(\mathrm{h}^{\mathrm{k}+\frac{1}{2}}\right)$ with polynomial of degree $k$ for nonlinear Burger's equation.

The outline of the paper is as follows. In Section 2, we will present the LDG method with generalized alternating numerical flux for the heat equation transformed from Burger's equation. Then the construction and analysis of the generalized Gauss-Radau projections are stated in Subsection 2.2. After that, we will give the stability and a priori error estimates in the $\mathrm{L}^{2}$-norm in Subsections 2.3 and 2.4. In Section 
3, we then move to the time discretization of semi-discrete form by the matrix exponential method. In section 4 , we present some numerical experiments that confirm our theoretical analysis.

\section{The LDG Method}

\subsection{Burger's equation}

Introducing the variable $w(x, t)$ and using the Hopf-Cole transformation

$$
u(x, t)=-2 v \frac{w_{x}(x, t)}{w(x, t)}
$$

we can derive that $w(x, t)$ satisfied the following equation

$$
\frac{\partial w}{\partial t}=v \frac{\partial^{2} w}{\partial x^{2}}
$$

By introducing a variable $p=\sqrt{v} w_{x}$ the Burger's equation (1.1) can be changed into the following system

$$
p=\sqrt{v} w_{x}, \quad w_{t}=\sqrt{v} p_{x}
$$

By this transformation the initial condition (1.2) becomes

$$
w(x, 0)=\exp \left\{-\int_{0}^{x} \frac{f(s)}{2 v} \mathrm{~d} s\right\}, \quad a<x<b,
$$

and the boundary condition (1.3) can be transformed into Neumann boundary conditions

$$
w_{x}(a, t)=0, \quad w_{x}(b, t)=0 .
$$

The computational domain $\Omega=[a, b]$ is divided into J cells $I_{j}=\left[x_{j-\frac{1}{2}}, x_{j+\frac{1}{2}}\right]$, for $1 \leqslant j \leqslant J$, in which:

$$
a=x_{\frac{1}{2}}<x_{\frac{3}{2}}<\cdots<x_{J+\frac{1}{2}}=b .
$$

Set $h_{j}=x_{j+\frac{1}{2}}-x_{j-\frac{1}{2}}$ and $h=\max _{j} h_{j}$. The associated finite element space is defined as piecewise polynomials space,

$$
V_{h}^{k}=\left\{v:\left.v\right|_{I_{j}} \in P^{k}\left(I_{j}\right), j=1, \ldots, J\right\},
$$

where $P^{k}\left(I_{j}\right)$ denotes the set of all polynomials of degree up to $k$ on $I_{j}$. The basis function in the space $V_{h}^{k}$ is chosen as Legendre polynomials. As we will see, this choice produce a diagonal mass matrix whose inversion can be easily performed. We map each element $I_{j}$ into the reference element $[-1,1]$ by introducing local coordinate $\xi \in[-1,1]$ such that $\xi=\frac{x-x_{j}}{h_{j} / 2}$. Now the basis function on element $I_{j}$ is chosen as $v_{m}^{j}(x)=P_{m}(\xi)$ for $j=1,2, \ldots, J$, where $P_{m}(\xi)$ is the rescaled Legendre polynomial of degree $m$ in $\xi$. With this coordinate transformation, the numerical solution in the space $V_{h}^{k}$ can be written as follows: $w_{h}(x, t)=\sum_{m=0}^{k} w_{j, m}(t) v_{j}^{m}(x)=\sum_{m=0}^{k} w_{j, m}(t) P^{m}(\xi)=\mathbf{V}^{\top}(x) \mathbf{W}_{j}(t)$, where $\mathbf{V}=\left(P_{0}(\xi), P_{1}(\xi), \ldots, P_{k}(\xi)\right)^{\top}$ denotes the Legendre basis functions, and $\mathbf{W}_{\mathbf{j}}(\mathrm{t})=\left(w_{j, 0}, w_{j, 1}, \ldots, w_{j, k}\right)^{\top}$ denotes the degrees of freedom (DOF) in cell $\mathrm{I}_{j}$. The global degrees of freedom in $\Omega$ is the set of $\mathbf{W}_{\mathbf{j}}(t)$ as $\mathbf{W}=\left[\mathbf{W}_{1} ; \mathbf{W}_{2} ; \ldots, \mathbf{W}_{\mathrm{J}}\right]$.

The $w_{j+\frac{1}{2}}^{+}$and $w_{j+\frac{1}{2}}^{-}$are values of $w$ at $x_{j+\frac{1}{2}}$ from the right cell $\mathrm{I}_{j+1}$ and the left cell $\mathrm{I}_{j}$, respectively,

$$
w_{j+\frac{1}{2}}^{+}=\lim _{\epsilon \rightarrow 0+} w\left(x_{j+\frac{1}{2}}+\epsilon\right), \quad w_{j+\frac{1}{2}}^{-}=\lim _{\epsilon \rightarrow 0+} w\left(x_{j+\frac{1}{2}}-\epsilon\right) .
$$


And at each element boundary point $x_{j+\frac{1}{2}}$ the jump term is denoted by

$$
\llbracket v \rrbracket_{j+\frac{1}{2}}=v_{j+\frac{1}{2}}^{+}-v_{j+\frac{1}{2}}^{-}
$$

and the weighted average is defined by

$$
v_{j+\frac{1}{2}}^{(\theta)}=\theta v_{j+\frac{1}{2}}^{-}+\widetilde{\theta} v_{j+\frac{1}{2}}^{+}, j=1,2, \ldots, J-1,
$$

where $\widetilde{\theta}=1-\theta$.

The aim of solving the system (2.3) with local discontinuous Galerkin method is to find $w_{h}, p_{h} \in V_{h}^{k}$ so that for all test functions $v_{h}, q_{h} \in V_{h}^{k}$ and all $1 \leqslant j \leqslant J$ are satisfied

$$
\begin{array}{r}
\int_{I_{j}} p_{h} q_{h} d x=-\sqrt{v} \int_{I_{j}} w_{h} q_{h x} d x+\left(\sqrt{v} \hat{w}_{h} q_{h}^{-}\right)_{j+\frac{1}{2}}-\left(\sqrt{v} \hat{w}_{h} q_{h}^{+}\right)_{j-\frac{1}{2}}, \\
\int_{I_{j}}\left(w_{h}\right)_{t} v_{h} d x=-\sqrt{v} \int_{I_{j}} p_{h} v_{h x} d x+\left(\sqrt{v} \hat{p}_{h} v_{h}^{-}\right)_{j+\frac{1}{2}}-\left(\sqrt{v} \hat{w}_{h} v_{h}^{+}\right)_{j-\frac{1}{2}},
\end{array}
$$

where the numerical fluxes $\hat{w}_{h}, \hat{p}_{h}$, for $j=1,2, \ldots, J-1$, are defined as follows

$$
\left.\hat{w}_{h}\right|_{j+\frac{1}{2}}=w_{h}^{(\theta)},\left.\quad \hat{p}_{h}\right|_{j+\frac{1}{2}}=p_{h}^{(\widetilde{\theta})},
$$

on two boundary points, due to the homogeneous Neumann boundary conditions, the numerical fluxes are defined as

$$
\left(\hat{w}_{\mathrm{h}}\right)_{\frac{1}{2}}=\left(w_{\mathrm{h}}\right)_{\frac{1}{2}}^{+}, \quad\left(\hat{w_{\mathrm{h}}}\right)_{\mathrm{J}+\frac{1}{2}}=\left(w_{\mathrm{h}}\right)_{\mathrm{J}+\frac{1}{2}}^{-}, \quad\left(\hat{p_{\mathrm{h}}}\right)_{\frac{1}{2}}=0, \quad\left(\hat{p_{\mathrm{h}}}\right)_{\mathrm{J}+\frac{1}{2}}=0 .
$$

\subsection{Generalized Gauss-Radau projection}

In order to obtain the optimal error estimates, here we select two generalized Gauss-Radau projections. For any function $z$ which is smooth enough, the generalized Gauss-Radau projection of $z, \mathrm{P}_{\theta} z$, is defined as the unique element in $\mathrm{P}^{\mathrm{k}}\left(\mathrm{I}_{j}\right)$ satisfying

$$
\begin{aligned}
\int_{\mathrm{I}_{j}} \mathrm{P}_{\theta} z v \mathrm{~d} x & =\int_{\mathrm{I}_{j}} z v \mathrm{~d} x, \quad \forall v \in \mathrm{P}^{k-1}\left(\mathrm{I}_{j}\right), \quad j=1,2, \ldots, \mathrm{J}, \\
\left(\mathrm{P}_{\theta} z\right)^{(\theta)}\left(x_{j+\frac{1}{2}}\right) & =z^{(\theta)}\left(x_{j+\frac{1}{2}}\right), \quad j=1,2, \ldots, \mathrm{J}-1, \\
\mathrm{P}_{\theta} z\left(x_{\mathrm{J}+\frac{1}{2}}^{-}\right) & =z\left(x_{\mathrm{J}+\frac{1}{2}}^{-}\right) .
\end{aligned}
$$

Similarly, $\mathrm{Q}_{\widetilde{\theta}} z$ is defined as

$$
\begin{aligned}
& \int_{\mathrm{I}_{\mathrm{j}}} \mathrm{Q}_{\tilde{\theta}} z \mathrm{q} \mathrm{d} x=\int_{\mathrm{I}_{\mathrm{j}}} z \mathrm{qd} x, \quad \forall \mathrm{q} \in \mathrm{P}^{\mathrm{k}-1}\left(\mathrm{I}_{\mathrm{j}}\right), \quad \mathrm{j}=1,2, \ldots, \mathrm{J}, \\
& \left(\mathrm{Q}_{\widetilde{\theta}} z\right)^{\widetilde{(\theta)}}\left(x_{j+\frac{1}{2}}\right)=z^{\widetilde{(\theta)}}\left(x_{j+\frac{1}{2}}\right), \quad j=1,2, \ldots, J-1, \\
& \mathrm{Q}_{\widetilde{\theta}} z\left(x_{\frac{1}{2}}^{+}\right)=z\left(x_{\frac{1}{2}}^{+}\right) \text {. }
\end{aligned}
$$

Under the definition of the above projections, we can obtain the following lemma.

Lemma 2.1. Let projection $\mathrm{Z}_{\mathrm{h}}$ be either $\mathrm{P}_{\theta}$ or $\mathrm{Q}_{\tilde{\theta}}$, and assume that the function $z$ is smooth enough. For $\theta \neq \frac{1}{2}$, the projection error $\eta=Z_{h} z-z$ satisfies

$$
\|\eta\|_{\mathrm{L}^{2}\left(\Omega_{h}\right)}+\mathrm{h}^{\frac{1}{2}}\|\eta\|_{\mathrm{L}^{2}\left(\Gamma_{\mathrm{h}}\right)} \leqslant \mathrm{Ch}^{\mathrm{k}+1}\|z\|_{W_{\infty}^{k+1}\left(\Omega_{h}\right)},
$$

where $\mathrm{C}>0$ is denoted a generic constant independent of $\mathrm{h}$ and $z$.

Proof. We introduce a projection $\pi_{h} z$ which is defined as the unique polynomial function in $\mathrm{P}^{\mathrm{k}}\left(\mathrm{I}_{\mathrm{j}}\right)$ satis- 
fying

$$
\int_{\mathrm{I}_{\mathrm{j}}} \pi_{\mathrm{h}} z v \mathrm{~d} x=\int_{\mathrm{I}_{\mathrm{j}}} z v \mathrm{~d} x, \quad \forall v \in \mathrm{P}^{\mathrm{k}}\left(\mathrm{I}_{\mathrm{j}}\right), \quad \mathrm{j}=1,2, \ldots, \mathrm{J} .
$$

It can be easily obtained that $\pi_{\mathrm{h}} z$ is uniquely defined and satisfies the optimal approximation

$$
\left\|z-\pi_{h} z\right\|_{L^{2}\left(I_{j}\right)}+h^{\frac{1}{2}}\left\|z-\pi_{h} z\right\|_{L^{\infty}\left(I_{j}\right)} \leqslant C h^{k+1}\|z\|_{W_{\infty}^{k+1}\left(I_{j}\right)}
$$

where $C>0$ is the bounding constant which is independent of $h$ and $z$.

Firstly, we consider the case that $\theta>\frac{1}{2}$. Take $Z_{h}=P_{\theta}$ and define $E=P_{\theta} z-\pi_{h} z$, we can obtain

$$
\begin{aligned}
\int_{\mathrm{I}_{\mathrm{j}}} \mathrm{E} v_{\mathrm{h}} \mathrm{d} x & =0, \quad \forall v_{\mathrm{h}} \in \mathrm{P}^{\mathrm{k}-1}\left(\mathrm{I}_{\mathrm{j}}\right), \quad j=1,2, \ldots, \mathrm{J}, \\
\mathrm{E}_{\mathrm{j}+\frac{1}{2}}^{(\theta)} & =\left(z-\pi_{\mathrm{h}} z\right)_{j+\frac{1}{2}}^{(\theta)} \equiv b_{j}, \quad j=1,2, \ldots, \mathrm{J}-1, \\
\mathrm{E}_{\mathrm{J}+\frac{1}{2}} & =\left(z-\pi_{\mathrm{h}} z\right)_{\mathrm{J}+\frac{1}{2}}^{-} \equiv \mathrm{b}_{\mathrm{J}} .
\end{aligned}
$$

We can derive that $\|\eta\|=\left\|z-\mathrm{P}_{\theta} z\right\|=\left\|z-\pi_{\mathrm{h}} z+\pi_{\mathrm{h}} z-\mathrm{P}_{\theta} z\right\| \leqslant\left\|z-\pi_{\mathrm{h}} z\right\|+\left\|\pi_{\mathrm{h}} z-\mathrm{P}_{\theta} z\right\|$. With the approximation (2.10), we can complete the proof by proving that $E$ is uniquely defined and satisfies the approximation property

$$
\|E\|_{L^{2}\left(\Omega_{h}\right)}+h^{\frac{1}{2}}\|E\|_{L^{2}\left(\Gamma_{h}\right)} \leqslant C h^{k+1}\|z\|_{W_{\infty}^{k+1}\left(\Omega_{h}\right)} .
$$

Due to $E(x) \in V_{h}^{k}$, it can be written as $E(x)=\sum_{m=0}^{k} \alpha_{j, m} v_{j}^{m}(x)=\sum_{m=0}^{k} \alpha_{j, m} P_{m}(\xi)$ where $P_{m}(\xi)$ is the rescaled Legendre polynomial of degree $m$ in $j$-th element. In this way, we can rewrite (2.11) as

$$
\int \sum_{I_{j}}^{k} \alpha_{j, m} P_{m}(\xi) \nu_{h} d x=0, \quad \forall v \in P^{k-1}\left(I_{j}\right) .
$$

Noticing the orthogonality of Legendre polynomials and $P_{k}( \pm 1)=( \pm 1)^{k}$ it is easy to get that

$$
E(x)=\alpha_{j, k} P_{k}(\xi) .
$$

According to (2.12)-(2.13) we have

$$
E_{j+\frac{1}{2}}^{(\theta)}=\theta \alpha_{j, k}+\widetilde{\theta}(-1)^{k} \alpha_{j+1, k}=b_{j}, \quad j=1 \cdots J-1 .
$$

Noticing the boundary condition (2.8) we can obtain the following forms of nonhomogeneous linear equations

$$
\left[\begin{array}{ccccc}
\theta & \widetilde{\theta}(-1)^{\mathrm{k}} & & \ldots & \\
& \theta & \widetilde{\theta}(-1)^{\mathrm{k}} & \ldots & \\
& & \ddots & & \\
& & & \theta & \widetilde{\theta}(-1)^{\mathrm{k}} \\
& & & & 1
\end{array}\right]\left[\begin{array}{c}
\alpha_{1, k} \\
\alpha_{2, \mathrm{k}} \\
\vdots \\
\alpha_{\mathrm{J}, \mathrm{k}}
\end{array}\right]=\left[\begin{array}{c}
\mathrm{b}_{1} \\
\mathrm{~b}_{2} \\
\vdots \\
\mathrm{b}_{\mathrm{J}}
\end{array}\right]
$$

We can write (2.14) into matrix formulation as $A_{\theta} \overrightarrow{\alpha_{J}}=\overrightarrow{b_{J}}$, where $\overrightarrow{b_{J}}=\left(b_{1}, b_{2}, \ldots, b_{J}\right)^{\top}$, and $\overrightarrow{\alpha_{J}}=$ $\left(\alpha_{1, k}, \alpha_{2, k}, \ldots, \alpha_{J, k}\right)^{\top}$. Since $\operatorname{det}\left(A_{\theta}\right)=\theta^{J-1}$, we can get a conclusion that both $E$ and the projection $Z_{h} z$ is uniquely defined provided $\theta \neq \frac{1}{2}$. After a simple operation, we can get

$$
A_{\theta}^{-1}=\frac{1}{\theta}\left[\begin{array}{cccccc}
1 & \zeta & \zeta^{2} & \cdots & \zeta^{J-2} & \theta \zeta^{J-1} \\
& 1 & \zeta & \cdots & \zeta^{J-3} & \theta \zeta^{J-2} \\
& & 1 & \cdots & \zeta^{J-4} & \theta \zeta^{J-3} \\
& & & \ddots & \vdots & \vdots \\
& & & & 1 & \theta \zeta \\
& & & & & \theta
\end{array}\right]
$$


where $\zeta=(-1)^{\mathrm{k}+1} \widetilde{\theta} \theta^{-1}$.

In this case,

$$
\alpha_{j, k}=\zeta^{J-j} b_{J}+\frac{1}{\theta} \sum_{m=j}^{J-1} b_{m} \zeta^{m-j} \leqslant \max \left(1, \theta^{-1}\right) \sum_{m=1}^{J} b_{m} \zeta^{m-j}, j=1,2, \ldots, J .
$$

For $Z_{h}=Q_{\widetilde{\theta}}$, taking the same processing method as mentioned above we have

$$
\alpha_{j, k}=(-1)^{k}\left(\zeta^{j-1} b_{1}+\frac{1}{\theta} \sum_{m=2}^{j} b_{m} \zeta^{j-m}\right) \leqslant \max \left(1, \theta^{-1}\right)(-1)^{k} \sum_{m=1}^{j} b_{m} \zeta^{j-m}, j=1,2, \ldots, J .
$$

No matter which projection is under, since $b_{j}$ is related to $L^{2}$ projection error, we can use the approximation property (2.10) to get

$$
\left|b_{m}\right| \leqslant C h^{k+\frac{1}{2}}\|z\|_{W_{\infty}^{k+1}\left(I_{j}\right)} .
$$

Since $\theta>\frac{1}{2}$, and then $|\zeta|=\left|\frac{\widetilde{\theta}}{\theta}\right| \leqslant 1$. It is easy to see that

$$
\begin{aligned}
\left|\alpha_{j, k}\right| & \leqslant C h^{k+1}\|z\|_{W_{\infty}^{k+1}\left(I_{j}\right)}\left(1+|\zeta|+\cdots+\left|\zeta^{J-1}\right|\right) \\
& \leqslant C h^{k+1}\|z\|_{W_{\infty}^{k+1}\left(I_{j}\right)} \frac{1-|\zeta|^{J}}{1-|\zeta|}=C(\theta) h^{k+\frac{1}{2}}\|z\|_{W_{\infty}^{k+1}\left(I_{j}\right)}
\end{aligned}
$$

and consequently

$$
\|\mathrm{E}\|_{\mathrm{L}^{2}\left(\Omega_{h}\right)}^{2}=\sum_{j=1}^{J}\left|\alpha_{j, k}\right|^{2}\left\|P_{j, k(x)}\right\|_{\mathrm{L}^{2}\left(\mathrm{I}_{j}\right)}^{2}=\sum_{j=1}^{J} \frac{h_{j} \alpha_{j, k}^{2}}{2 k+1} \leqslant \mathrm{Ch}\left\|\overrightarrow{\alpha_{J}}\right\|_{2}^{2}, \quad\|\mathrm{E}\|_{\mathrm{L}^{2}\left(\Gamma_{h}\right)}^{2}=\sum_{j=1}^{J}\left|\alpha_{j, k}\right|^{2}=\left\|\overrightarrow{\alpha_{J}}\right\|_{2}^{2},
$$

where $\left\|\overrightarrow{\alpha_{J}}\right\|_{2}^{2}=\sum_{j=1}^{J}\left|\alpha_{j, k}\right|^{2} \leqslant \mathrm{Ch}^{2 \mathrm{k}+1}$.

Then we continue to consider the other case that $\theta<\frac{1}{2}$. In this case we can choose $Z_{h}=P_{\widetilde{\theta}}$ and get the same conclusion. Finally we complete the proof.

Remark 2.2. For $\theta=\frac{1}{2}$, in other word $\zeta=1$ in (2.16), it is apparent that in this case $C(\theta)=\mathrm{O}\left(\frac{1}{h}\right)$ and can only get the sub-optimal convergence results of order $k$. However, if $k$ is even and the mesh is uniform, we can obtain the optimal approximation by using the super-convergence property. For more details, we refer to [9].

\subsection{Stability analysis}

Considering the boundary conditions and the definition of the jump term, we sum up (2.5)-(2.6) on the whole area

$$
\begin{aligned}
& \int_{\Omega} p_{h} q_{h} d x=-\sqrt{v} \sum_{j=1}^{J} \int_{I_{j}} w_{h} q_{h x} d x-\sqrt{v} \sum_{j=0}^{J-1} \hat{w}_{h} \llbracket q_{h} \rrbracket_{j+\frac{1}{2}}+\sqrt{v}\left(q_{h}^{-} w_{h}^{-}\right)_{J+\frac{1}{2}}-\sqrt{v}\left(q_{h}^{+} w_{h}^{+}\right)_{\frac{1}{2}}, \\
& \int_{\Omega}\left(w_{h}\right)_{t} v_{h} d x=-\sqrt{v} \sum_{j=1}^{J} \int_{I_{j}} p_{h} v_{h x} d x-\sqrt{v} \sum_{j=1}^{J-1} \hat{p}_{h} \llbracket v_{h} \rrbracket_{j+\frac{1}{2}} \cdot
\end{aligned}
$$

Next we add on the above two equations to get

$$
\int_{\Omega} p_{h} q_{h} d x+\int_{\Omega}\left(w_{h}\right)_{t} v_{h} d x+\sqrt{v} B\left(w_{h}, v_{h}, p_{h}, q_{h}\right)=0,
$$


where

$$
\begin{aligned}
\mathrm{B}\left(w_{h}, v_{h}, p_{h}, q_{h}\right)= & \sum_{j=1}^{J} \int_{I_{j}} w_{h} q_{h x} d x+\sum_{j=1}^{J} \int_{I_{j}} p_{h} v_{h x} d x \\
& +\sum_{j=1}^{J-1} \hat{w}_{h} \llbracket q_{h} \rrbracket_{j+\frac{1}{2}}+\sum_{j=1}^{J-1} \hat{p_{h}} \llbracket v_{h} \rrbracket_{j+\frac{1}{2}}-\left(q_{h}^{-} w_{h}^{-}\right)_{J+\frac{1}{2}}+\left(q_{h}^{+} w_{h}^{+}\right)_{\frac{1}{2}} .
\end{aligned}
$$

Theorem 2.3. The solution of LDG scheme (2.5)-(2.6) is stable in the $\mathrm{L}^{2}$-norm, namely the numerical solution $w_{\mathrm{h}}$ satisfies

$$
\left\|w_{\mathrm{h}}(\cdot, \mathrm{T})\right\| \leqslant\left\|w_{\mathrm{h}}(\cdot, 0)\right\| .
$$

Proof. Taking $v_{\mathrm{h}}=w_{\mathrm{h}}, \mathrm{q}_{\mathrm{h}}=\mathrm{p}_{\mathrm{h}}$ into (2.18), we have

$$
\begin{aligned}
\mathrm{B}\left(w_{\mathrm{h}}, w_{h}, p_{h}, p_{h}\right)= & \sum_{j=1}^{J} \int_{\mathrm{I}_{j}} w_{h} p_{h x} \mathrm{~d} x+\sum_{j=1}^{J} \int_{\mathrm{I}_{j}} p_{h} w_{h x} \mathrm{dx} \\
& +\sum_{j=1}^{J-1} \hat{w}_{\mathrm{h}} \llbracket p_{h} \rrbracket_{j+\frac{1}{2}}+\sum_{j=1}^{J-1} \hat{p_{h}} \llbracket w_{h} \rrbracket_{j+\frac{1}{2}}-\left(p_{h}^{-} w_{h}^{-}\right)_{J+\frac{1}{2}}+\left(p_{h}^{+} w_{h}^{+}\right)_{\frac{1}{2}} .
\end{aligned}
$$

Taking partial integration for the first term on the right hand side to obtain

$$
\sum_{j=1}^{J} \int_{I_{j}} w_{h} p_{h x} d x=-\sum_{j=1}^{J} \int_{I_{j}} p_{h} w_{h x} d x-\sum_{j=1}^{J-1}\left(p_{h}^{+} w_{h}^{+}-p_{h}^{-} w_{h}^{-}\right)_{j+\frac{1}{2}}-\left(p_{h}^{+} w_{h}^{+}\right)_{\frac{1}{2}}+\left(p_{h}^{-} w_{h}^{-}\right)_{J+\frac{1}{2}} .
$$

Using the definition of the numerical flux (2.7) we obtain

$$
\begin{aligned}
\sum_{j=1}^{J-1} \hat{w}_{h} \llbracket p_{h} \rrbracket_{j+\frac{1}{2}}+\sum_{j=1}^{J-1} \hat{p_{h} \llbracket w_{h} \rrbracket_{j+\frac{1}{2}}} & =\sum_{j=1}^{J-1}\left\{\left(\theta w_{h}^{-}+\widetilde{\theta} w_{h}^{+}\right) \llbracket p_{h} \rrbracket+\left(\theta p_{h}^{-}+\widetilde{\theta} p_{h}^{+}\right) \llbracket w_{h} \rrbracket\right\}_{j+\frac{1}{2}} \\
& =\sum_{j=1}^{J-1}\left\{\theta\left(w_{h}^{-} \llbracket p_{h} \rrbracket+p_{h}^{+} \llbracket w_{h} \rrbracket\right)+\widetilde{\theta}\left(w_{h}^{+} \llbracket p_{h} \rrbracket+p_{h}^{-} \llbracket w_{h} \rrbracket\right\}_{j+\frac{1}{2}}\right. \\
& =\sum_{j=1}^{J-1}\left[\theta\left(w_{h}^{+} P_{h}^{+}-w_{h}^{-} p_{h}^{-}\right)+\widetilde{\theta}\left(w_{h}^{+} P_{h}^{+}-w_{h}^{-} p_{h}^{-}\right)\right]_{j+\frac{1}{2}} \\
& =\sum_{j=1}^{J-1}\left(w_{h}^{+} p_{h}^{+}-w_{h}^{-} p_{h}^{-}\right)_{j+\frac{1}{2}} .
\end{aligned}
$$

In the above derivation we have used the following two formulas

$$
\begin{aligned}
& \sum_{j=1}^{J-1}\left(p_{h}^{+} w_{h}^{+}-p_{h}^{-} w_{h}^{-}\right)_{j+\frac{1}{2}}=\sum_{j=1}^{J-1}\left(p_{h}^{+} w_{h}^{+}-p_{h}^{+} w_{h}^{-}+p_{h}^{+} w_{h}^{-}-p_{h}^{-} w_{h}^{-}\right)_{j+\frac{1}{2}} \sum_{j=1}^{J-1}\left(p_{h}^{+} \llbracket w_{h} \rrbracket+w_{h}^{-} \llbracket p_{h} \rrbracket\right)_{j+\frac{1}{2}} \\
& \sum_{j=1}^{J-1}\left(p_{h}^{+} w_{h}^{+}-p_{h}^{-} w_{h}^{-}\right)_{j+\frac{1}{2}}=\sum_{j=1}^{J-1}\left(p_{h}^{+} w_{h}^{+}-w_{h}^{+} p_{h}^{-}+w_{h}^{+} p_{h}^{-}-p_{h}^{-} w_{h}^{-}\right)_{j+\frac{1}{2}}=\sum_{j=1}^{J-1}\left(w_{h}^{+} \llbracket p_{h} \rrbracket+p_{h}^{-} \llbracket w_{h} \rrbracket\right)_{j+\frac{1}{2}} .
\end{aligned}
$$

Substituting (2.20) and (2.21) into (2.19) we can draw the conclusion

$$
\mathrm{B}\left(w_{\mathrm{h}}, w_{\mathrm{h}}, \mathrm{p}_{\mathrm{h}}, \mathrm{p}_{\mathrm{h}}\right)=0
$$


Applying this conclusion, (2.17) becomes

$$
\int_{\Omega} p_{h}^{2} d x+\int_{\Omega}\left(w_{h}\right)_{t} w_{h} d x=0
$$

i.e.,

In other word, it is true that

$$
\frac{1}{2} \frac{\mathrm{d}}{\mathrm{dt}}\left\|w_{\mathrm{h}}\right\|^{2}+\left\|p_{\mathrm{h}}\right\|^{2}=0
$$

This finishes the proof of stability analysis.

$$
\frac{\mathrm{d}}{\mathrm{dt}}\left\|w_{\mathrm{h}}\right\|^{2} \leqslant 0
$$

\subsection{Error estimate}

In this subsection, a priori error estimates in the $\mathrm{L}^{2}$-norm for LDG scheme (2.5)-(2.6) will be presented by virtue of the generalized Gauss-Radau projection and stability analysis.

Theorem 2.4. Assume the exact solution of (2.2) is smooth and $\theta \neq \frac{1}{2}$. The numerical solution $w_{\mathrm{h}}$ of the Burgers equation obtained by (2.5)-(2.6) with exact solution $w$ satisfies the following error estimates

$$
\left\|w(x, t)-w_{h}(x, t)\right\|^{2} \leqslant \mathrm{Ch}^{2 \mathrm{k}+1} .
$$

Proof. First, we represent the error terms as follows by introducing new alternative notations

$$
\begin{aligned}
e_{w} & =w-w_{h}=\left(w-P_{\theta} w\right)+\left(P_{\theta} w-w_{h}\right)=\eta_{w}-\varepsilon_{w}, \\
e_{p} & =p-p_{h}=\left(p-Q_{\theta} p\right)+\left(Q_{\theta} p-p_{h}\right)=\eta_{p}-\varepsilon_{p} .
\end{aligned}
$$

By Lemma 2.1 we can see that

$$
\left\|\eta_{w}\right\|_{L^{2}\left(\Omega_{h}\right)}+h^{\frac{1}{2}}\left\|\eta_{w}\right\|_{L^{2}\left(\Gamma_{h}\right)} \leqslant C h^{k+1}\|w\|_{k+1, \Omega_{h}} .
$$

So the follows we just need to prove that $\left\|\varepsilon_{w}\right\|_{\mathrm{L}^{2}\left(\Omega_{h}\right)} \leqslant \mathrm{Ch}^{2 \mathrm{k}+1}\|w\|_{k+1, \Omega_{h}}$. For the exact solutions it is true that

$$
\int_{\Omega} p q_{h} \mathrm{~d} x+\int_{\Omega}(w)_{t} v_{h} d x+\sqrt{v} B\left(w, v_{h}, p, q_{h}\right)=0
$$

Noticing (2.17) and the representation of $e_{w}, e_{p}$, we can obtain that

$$
\int_{\Omega}\left(\eta_{p}-\varepsilon_{p}\right) q_{h} d x+\int_{\Omega}\left(\eta_{w}-\varepsilon_{w}\right)_{t} v_{h} d x+\sqrt{v} B\left(\eta_{w}-\varepsilon_{w}, v_{h}, \eta_{p}-\varepsilon_{p}, q_{h}\right)=0 .
$$

Taking $v_{\mathrm{h}}=\varepsilon_{w}, \mathrm{q}_{\mathrm{h}}=\varepsilon_{\mathrm{p}}$ into (2.24), we get

$$
\begin{aligned}
& \int_{\Omega} \varepsilon_{p}^{2} d x+\int_{\Omega}\left(\varepsilon_{w}\right)_{t} \varepsilon_{w} d x+\sqrt{v} B\left(\varepsilon_{w}, \varepsilon_{w}, \varepsilon_{p}, \varepsilon_{p}\right) \\
& =\int_{\Omega} \eta_{p} \varepsilon_{p} d x+\int_{\Omega}\left(\eta_{w}\right)_{t} \varepsilon_{w} d x+\sqrt{v} B\left(\eta_{w}, \varepsilon_{w}, \eta_{p}, \varepsilon_{p}\right) .
\end{aligned}
$$

According to the stability result (2.22), the left hand side of (2.25) can be rewritten into

$$
\text { LHS }=\frac{1}{2} \frac{\mathrm{d}}{\mathrm{dt}}\left\|\varepsilon_{w}\right\|^{2}+\left\|\varepsilon_{\mathrm{p}}\right\|^{2} .
$$

Noticing the definition of $\mathrm{P}_{\theta} \mathcal{w}, \mathrm{Q}_{\theta} \mathrm{p}$, we have that

$$
\int_{\mathrm{I}_{j}} \eta_{w}\left(\varepsilon_{\mathfrak{p}}\right)_{x} \mathrm{~d} x=0, \quad \int_{\mathrm{I}_{\mathrm{j}}} \eta_{\mathfrak{p}}\left(\varepsilon_{w}\right)_{x} \mathrm{~d} x=0,
$$

because $q_{h x}$ and $v_{h x}$ are polynomials of degree at most $k-1$. It is easy to see that

$$
\left.\hat{\eta_{\hat{w}}}\right|_{j+\frac{1}{2}}=0,\left.\quad \hat{\eta_{p}}\right|_{j+\frac{1}{2}}=0
$$

at interior boundary points and 


$$
\left(\eta_{w}\right)_{\mathrm{J}+\frac{1}{2}}=0, \quad\left(\eta_{\mathrm{p}}\right)_{\frac{1}{2}}=0
$$

on boundary points.

In this case for the right hand side of (2.25), we can obtain

$$
\operatorname{RHS}=\int_{\Omega} \eta_{p} \varepsilon_{p} d x+\int_{\Omega}\left(\eta_{w}\right)_{t} \varepsilon_{w} d x+\sqrt{v}\left(\varepsilon_{p}^{+} \eta_{w}^{+}\right)_{\frac{1}{2}}
$$

It is easy to verify that

$$
\text { RHS } \leqslant \frac{d}{d t}\left\|\eta_{w}\right\|\left\|\varepsilon_{w}\right\|+\left\|\eta_{p}\right\|\left\|\varepsilon_{p}\right\|+\left|S_{1}\right|,
$$

where $\left|S_{1}\right|=\left|\varepsilon_{p}^{+}\right|_{\frac{1}{2}}\left|\eta_{w}^{+}\right|_{\frac{1}{2}}$. By using (2.9), Cauchy-Schwarz inequality, and Young's inequality, $\left|S_{1}\right|$ is bounded by

$$
\left|S_{1}\right| \leqslant C \sum_{j=1}^{J} h^{k+\frac{1}{2}}\|w\|_{\infty}^{k+1}\left(I_{j}\right)\left\|\varepsilon_{\mathfrak{p}}\right\|_{L_{2}\left(I_{1}\right)} \leqslant C h^{2 k+1}+\frac{1}{4}\left\|\varepsilon_{\mathfrak{p}}\right\|^{2} .
$$

Similarly, for the right hand side, we apply the approximation results (2.9), Cauchy-Schwarz inequality, and Young's inequality to get

$$
\mathrm{RHS} \leqslant \mathrm{Ch}^{2 \mathrm{k}+2}+\left\|\varepsilon_{w}\right\|^{2}+\frac{1}{2}\left\|\varepsilon_{\mathrm{p}}\right\|^{2}+\left|\mathrm{S}_{1}\right| .
$$

Based on (2.26) and (2.27), we can rewritten (2.25) as follows

$$
\frac{\mathrm{d}}{\mathrm{dt}}\left\|\varepsilon_{\mathcal{w}}\right\|^{2}+\frac{1}{2}\left\|\varepsilon_{\mathfrak{p}}\right\|^{2} \leqslant \mathrm{Ch}^{2 \mathrm{k}+2}+\mathrm{Ch}^{2 \mathrm{k}+1}+2\left\|\varepsilon_{\mathcal{w}}\right\|^{2} .
$$

A Gronwall's inequality, the approximation results (2.9), and the initial error finally give us the error estimate (2.23).

Table 1: The $\mathrm{L}^{2}$ spatial errors and order of convergence for $v=0.1$.

\begin{tabular}{|cc|cccccc|}
\hline \multirow{2}{*}{$\mathrm{m}$} & \multicolumn{2}{|c}{$\theta=0.2$} & \multicolumn{2}{c}{$\theta=0.5$} & \multicolumn{2}{c|}{$\theta=1.0$} \\
\cline { 3 - 8 } & & error & order & error & order & error & order \\
\hline \multirow{3}{*}{$\mathrm{P}^{0}$} & 3 & $3.49 \mathrm{E}-2$ & - & $2.54 \mathrm{E}-2$ & - & $4.49 \mathrm{E}-2$ & - \\
& 4 & $1.71 \mathrm{E}-2$ & 1.03 & $1.25 \mathrm{E}-2$ & 1.02 & $2.26 \mathrm{E}-2$ & 0.99 \\
& 5 & $8.48 \mathrm{E}-3$ & 1.01 & $6.23 \mathrm{E}-3$ & 1.01 & $1.13 \mathrm{E}-2$ & 1.00 \\
& 6 & $4.22 \mathrm{E}-3$ & 1.00 & $3.11 \mathrm{E}-3$ & 1.00 & $5.66 \mathrm{E}-3$ & 1.00 \\
\hline & 3 & $1.24 \mathrm{E}-2$ & - & $6.28 \mathrm{E}-3$ & - & $1.46 \mathrm{E}-2$ & - \\
$\mathrm{P}^{1}$ & 4 & $4.62 \mathrm{E}-3$ & 1.43 & $3.12 \mathrm{E}-3$ & 1.01 & $5.25 \mathrm{E}-3$ & 1.47 \\
& 5 & $1.65 \mathrm{E}-3$ & 1.49 & $1.56 \mathrm{E}-3$ & 1.00 & $1.86 \mathrm{E}-3$ & 1.50 \\
& 6 & $5.84 \mathrm{E}-4$ & 1.50 & $7.79 \mathrm{E}-4$ & 1.00 & $6.59 \mathrm{E}-4$ & 1.50 \\
\hline & 3 & $7.10 \mathrm{E}-5$ & - & $4.06 \mathrm{E}-5$ & - & $1.56 \mathrm{E}-4$ & - \\
$\mathrm{P}^{2}$ & 4 & $7.64 \mathrm{E}-6$ & 3.22 & $4.98 \mathrm{E}-6$ & 3.03 & $1.51 \mathrm{E}-5$ & 3.37 \\
& 5 & $8.74 \mathrm{E}-7$ & 3.13 & $6.19 \mathrm{E}-7$ & 3.01 & $1.52 \mathrm{E}-6$ & 3.31 \\
& 6 & $1.04 \mathrm{E}-7$ & 3.07 & $7.73 \mathrm{E}-8$ & 3.00 & $1.62 \mathrm{E}-7$ & 3.23 \\
\hline & 3 & $1.94 \mathrm{E}-5$ & - & $4.97 \mathrm{E}-6$ & - & $2.31 \mathrm{E}-5$ & - \\
$\mathrm{P}^{3}$ & 4 & $1.82 \mathrm{E}-6$ & 3.41 & $6.19 \mathrm{E}-7$ & 3.00 & $2.08 \mathrm{E}-6$ & 3.47 \\
& 5 & $1.63 \mathrm{E}-7$ & 3.48 & $7.70 \mathrm{E}-8$ & 3.00 & $1.80 \mathrm{E}-7$ & 3.49 \\
& 6 & $1.45 \mathrm{E}-8$ & 3.50 & $1.00 \mathrm{E}-8$ & 3.00 & $2.00 \mathrm{E}-8$ & 3.50 \\
\hline
\end{tabular}


Table 2: The $\mathrm{L}^{2}$ spatial errors and order of convergence for $v=0.01$.

\begin{tabular}{|c|c|c|c|c|c|c|c|}
\hline & \multirow{2}{*}{$\mathrm{m}$} & \multicolumn{2}{|c|}{$\theta=0.2$} & \multicolumn{2}{|c|}{$\theta=0.5$} & \multicolumn{2}{|c|}{$\theta=1.0$} \\
\hline & & error & order & error & order & error & order \\
\hline \multirow{4}{*}{$\mathrm{P}^{0}$} & 3 & $9.60 \mathrm{E}-3$ & - & $7.00 \mathrm{E}-3$ & - & $1.33 \mathrm{E}-2$ & - \\
\hline & 4 & 4.90E-3 & 0.99 & $3.50 \mathrm{E}-3$ & 1.00 & $6.70 \mathrm{E}-3$ & 0.99 \\
\hline & 5 & $2.40 \mathrm{E}-3$ & 1.00 & $1.70 \mathrm{E}-3$ & 1.00 & $3.30 \mathrm{E}-3$ & 1.00 \\
\hline & 6 & $1.20 \mathrm{E}-3$ & 1.00 & $9.00 \mathrm{E}-4$ & 1.00 & $1.70 \mathrm{E}-3$ & 1.00 \\
\hline \multirow{4}{*}{$p^{1}$} & 3 & $2.50 \mathrm{E}-3$ & - & $4.30 \mathrm{E}-3$ & - & $5.20 \mathrm{E}-3$ & - \\
\hline & 4 & $9.00 \mathrm{E}-4$ & 1.45 & $2.20 \mathrm{E}-3$ & 1.00 & $1.90 \mathrm{E}-3$ & 1.46 \\
\hline & 5 & $3.00 \mathrm{E}-4$ & 1.49 & $1.10 \mathrm{E}-3$ & 1.00 & $7.00 \mathrm{E}-4$ & 1.49 \\
\hline & 6 & $1.00 \mathrm{E}-4$ & 1.50 & $5.00 \mathrm{E}-4$ & 1.00 & $2.00 \mathrm{E}-4$ & 1.50 \\
\hline \multirow{4}{*}{$p^{2}$} & 3 & $1.94 \mathrm{E}-5$ & - & $1.14 \mathrm{E}-5$ & - & $5.45 \mathrm{E}-5$ & - \\
\hline & 4 & 2.17E-6 & 3.16 & $1.40 \mathrm{E}-6$ & 3.03 & $5.27 \mathrm{E}-6$ & 3.37 \\
\hline & 5 & $2.60 \mathrm{E}-7$ & 3.10 & $1.70 \mathrm{E}-7$ & 3.01 & $5.20 \mathrm{E}-7$ & 3.33 \\
\hline & 6 & $3.00 \mathrm{E}-8$ & 3.04 & $2.00 \mathrm{E}-8$ & 3.00 & $5.00 \mathrm{E}-8$ & 3.26 \\
\hline \multirow{4}{*}{$p^{3}$} & 3 & $3.88 \mathrm{E}-6$ & - & $1.51 \mathrm{E}-6$ & - & $8.20 \mathrm{E}-6$ & - \\
\hline & 4 & $3.62 \mathrm{E}-7$ & 3.42 & $1.86 \mathrm{E}-7$ & 3.03 & 7.50E-7 & 3.45 \\
\hline & 5 & $3.20 \mathrm{E}-8$ & 3.48 & $2.30 \mathrm{E}-8$ & 3.01 & $6.70 \mathrm{E}-8$ & 3.49 \\
\hline & 6 & 3.00E-9 & 3.50 & $3.00 \mathrm{E}-9$ & 3.00 & $6.00 \mathrm{E}-9$ & 3.50 \\
\hline
\end{tabular}

Table 3: The $\mathrm{L}^{2}$ spatial errors and order of convergence for $v=0.001$.

\begin{tabular}{|cc|cccccc|}
\hline \multirow{2}{*}{} & \multirow{2}{*}{$\mathrm{m}$} & \multicolumn{2}{|c}{$\theta=0.2$} & \multicolumn{2}{c}{$\theta=0.5$} & \multicolumn{2}{c|}{$\theta=1.0$} \\
\cline { 3 - 8 } $\mathrm{P}^{0}$ & & error & order & error & order & error & order \\
\hline & 3 & $1.10 \mathrm{E}-3$ & - & $8.00 \mathrm{E}-4$ & - & $1.50 \mathrm{E}-3$ & - \\
& 4 & $6.00 \mathrm{E}-4$ & 0.98 & $4.00 \mathrm{E}-4$ & 1.00 & $8.00 \mathrm{E}-4$ & 0.99 \\
& 5 & $3.00 \mathrm{E}-4$ & 0.99 & $2.00 \mathrm{E}-4$ & 1.00 & $4.00 \mathrm{E}-4$ & 1.00 \\
& 6 & $1.00 \mathrm{E}-4$ & 1.00 & $1.00 \mathrm{E}-4$ & 1.00 & $2.00 \mathrm{E}-4$ & 1.00 \\
\hline & 3 & $4.18 \mathrm{E}-4$ & - & $7.98 \mathrm{E}-4$ & - & $6.42 \mathrm{E}-4$ & - \\
$\mathrm{P}^{1}$ & 4 & $1.01 \mathrm{E}-4$ & 2.05 & $4.06 \mathrm{E}-4$ & 0.98 & $2.26 \mathrm{E}-4$ & 1.50 \\
& 5 & $3.50 \mathrm{E}-5$ & 1.53 & $2.04 \mathrm{E}-4$ & 0.99 & $7.97 \mathrm{E}-5$ & 1.50 \\
& 6 & $1.23 \mathrm{E}-5$ & 1.51 & $1.02 \mathrm{E}-4$ & 1.00 & $2.82 \mathrm{E}-5$ & 1.50 \\
\hline & 3 & $2.01 \mathrm{E}-6$ & - & $1.27 \mathrm{E}-6$ & - & $7.34 \mathrm{E}-6$ & - \\
$\mathrm{P}^{2}$ & 4 & $2.50 \mathrm{E}-7$ & 3.00 & $1.59 \mathrm{E}-7$ & 2.99 & $6.26 \mathrm{E}-7$ & 3.55 \\
& 5 & $3.00 \mathrm{E}-8$ & 3.01 & $2.00 \mathrm{E}-8$ & 3.00 & $6.20 \mathrm{E}-8$ & 3.34 \\
& 6 & $4.00 \mathrm{E}-9$ & 3.04 & $2.00 \mathrm{E}-9$ & 3.00 & $6.00 \mathrm{E}-9$ & 3.26 \\
\hline & 3 & $4.24 \mathrm{E}-7$ & - & $8.54 \mathrm{E}-7$ & - & $9.77 \mathrm{E}-7$ & - \\
$\mathrm{P}^{3}$ & 4 & $3.85 \mathrm{E}-8$ & 3.46 & $1.12 \mathrm{E}-7$ & 2.93 & $8.86 \mathrm{E}-8$ & 3.46 \\
& 5 & $3.40 \mathrm{E}-9$ & 3.48 & $1.42 \mathrm{E}-8$ & 3.00 & $7.90 \mathrm{E}-9$ & 3.49 \\
& 6 & $3.00 \mathrm{E}-10$ & 3.50 & $1.80 \mathrm{E}-9$ & 3.00 & $7.00 \mathrm{E}-10$ & 3.50 \\
\hline
\end{tabular}

Table 4: Comparison of the numerical results by LDG with exact and other numerical solutions in Example 4.2 for $v=0.1$ and $v=0.01$.

\begin{tabular}{|l|l|l|l|l|l|}
\hline \multicolumn{7}{|c|}{$v=0.1$} \\
\hline Method & $\mathrm{x}=0.1$ & $\mathrm{x}=0.3$ & $\mathrm{x}=0.5$ & $\mathrm{x}=0.7$ & $\mathrm{x}=0.9$ \\
\hline IFDM & 0.02909 & 0.08044 & 0.10939 & 0.09838 & 0.04037 \\
BEM & 0.02913 & 0.07951 & 0.10770 & 0.09663 & 0.03976 \\
LDG & 0.02876 & 0.07946 & 0.10789 & 0.09685 & 0.03969 \\
Exact & 0.02876 & 0.07946 & 0.10789 & 0.09685 & 0.03969 \\
\hline \multicolumn{7}{|c|}{$v=0.01$} \\
\hline Method & $\mathrm{x}=0.1$ & $\mathrm{x}=0.3$ & $\mathrm{x}=0.5$ & $\mathrm{x}=0.7$ & $\mathrm{x}=0.9$ \\
\hline IFDM & 0.04367 & 0.13095 & 0.21800 & 0.30466 & 0.38024 \\
BEM & 0.04300 & 0.12877 & 0.21468 & 0.30075 & 0.37452 \\
LDG & 0.04296 & 0.12884 & 0.21455 & 0.29998 & 0.37323 \\
Exact & 0.04296 & 0.12884 & 0.21456 & 0.30000 & 0.37328 \\
\hline
\end{tabular}




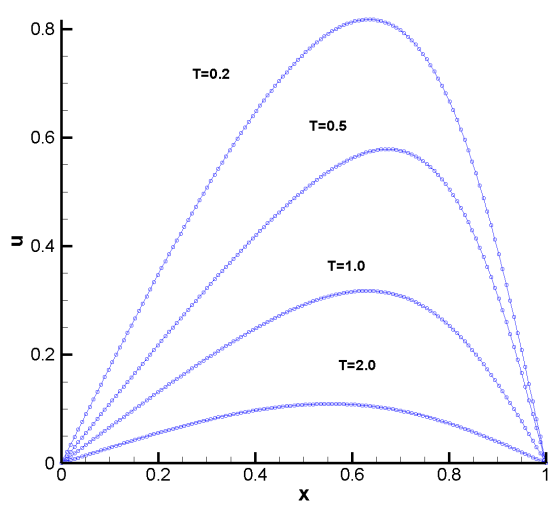

(a)

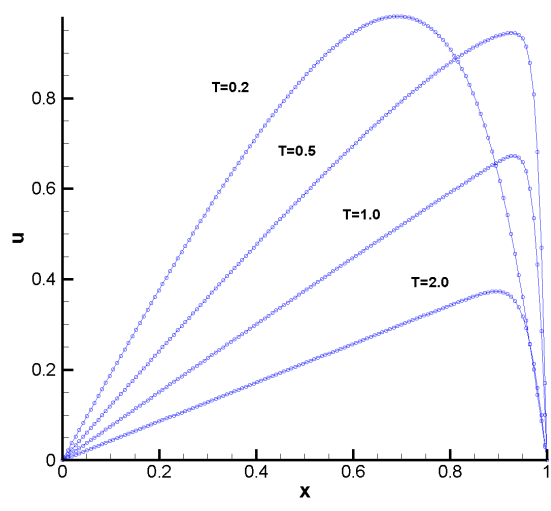

(b)

Figure 1: Numerical solutions at different times $T=0.2,0.5,1.0$, and 2.0 for (a) $v=0.1$, (b) 0.01 .

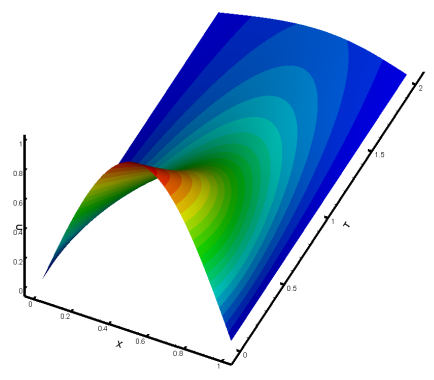

(a)

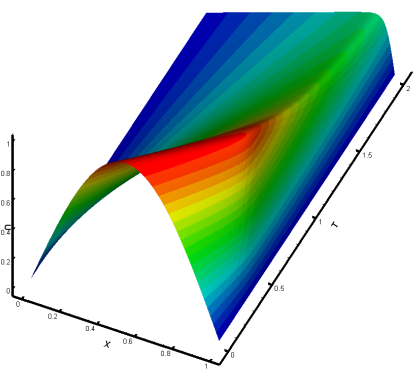

(b)

Figure 2: Numerical solution profile for Example 4.2: (a) $v=0.1$, (b) $v=0.01$.

\section{Time discretization}

So far, we have only studied the spatial discretization and left the time variable continuous. In this section, we will semi-discrete the formulation analytically by matrix exponential method. The LDG scheme (2.3) can be rewritten into the matrix equation of the form

$$
\begin{aligned}
\mathbf{M}_{1} \mathbf{P} & =\mathbf{A}_{1} \mathbf{W}, \\
\mathbf{M}_{2} \frac{\mathrm{d} \mathbf{W}}{\mathrm{dt}} & =\mathbf{A}_{2} \mathbf{P},
\end{aligned}
$$

where $\mathbf{W}$ and $\mathbf{P}$ are solution vectors containing the degrees of freedom (DOF) of $w_{h}$ and $p_{h}$. Since the mass matrices $\mathbf{M}_{1}$ and $\mathbf{M}_{2}$ are block diagonal, we can invert them easily. Solving $\mathbf{P}$ from (3.1), $\mathbf{P}=\mathbf{M}_{1}^{-1} \mathbf{A}_{1} \mathbf{U}$, substituting it into (3.2), and considering the initial condition (2.4), we obtain the following linear homogeneous ODE system:

$$
\begin{aligned}
& \frac{\mathrm{d} \mathbf{W}}{\mathrm{dt}}=\mathbf{A} \mathbf{W}, \mathrm{t} \in[0, \mathrm{~T}], \\
& \mathbf{W}(0)=\mathbf{W}_{0},
\end{aligned}
$$


where $\mathbf{A}=-\mathbf{M}_{2}^{-1} \mathbf{A}_{2}\left(\mathbf{M}_{1}^{-1} \mathbf{A}_{1}\right)$.

Assume the final time is $t=T$ and let time step $\triangle t=T / N, t_{n}=n \triangle t, 0 \leqslant n \leqslant N$. We multiply (3.3) by the integration factor $e^{-A t}$ and integrate over one time step from $t_{n}$ to $t_{n+1}$ to obtain

$$
\mathbf{W}^{\mathrm{n}+1}=\mathrm{e}^{\mathbf{A} \triangle \mathrm{t}} \mathbf{W}^{\mathrm{n}}, \mathrm{n}=0,1, \ldots, \mathrm{N}-1 .
$$

We can compute $\mathbf{U}$, the DOF of the solution of original Burger's equation $\mathfrak{u}_{h}$ as

$$
\mathbf{U}^{\mathrm{n}}=-2 \sqrt{v} \frac{\mathbf{P}^{\mathrm{n}}}{\mathbf{W}^{\mathrm{n}}}=-2 \sqrt{v} \frac{\mathbf{M}_{1}^{-1} \mathbf{A}_{1} \mathbf{U}^{\mathrm{n}}}{\mathbf{W}^{\mathrm{n}}}, \mathrm{n}=1,2, \ldots
$$

by using the Hopf-Cole transformation (2.1) and equation (2.3).

Remark 3.1. For one-dimensional case in this paper, the exponential of the square matrix $e^{\mathbf{A} \triangle t}$ is computed by using a scaling and squaring algorithm with a Pade approximation as implemented in "expm" of Matlab. For high dimensional problems, the calculation of matrix exponential will encounter tremendous difficulty because the exponential matrix of $\mathbf{A}$ is large and dense. This difficulty can be solved by using Krylov subspace method to approximate the products of the exponential matrix and vector. The reader is referred to consult the paper [8] for more details.

\section{Numerical experiments}

In this section, the solutions of the Burger's equation will be investigated by using the proposed LDG method with generalized alternating numerical flux. The LDG method is carried out with the piecewise polynomials of degree at most $k$ in spatial variable with $k=0,1,2,3$. The time step is chosen small enough such that the overall error is dominated by the spatial error. The computational space is divided into J elements to get the uniform mesh.

To show the error estimates of our method, we define the convergence order of the LDG method as

$$
\operatorname{order}_{\mathrm{J}}=\left(\log \left(\operatorname{error}_{\mathrm{J} / 2}\right)-\log \left(\text { error }_{\mathrm{J}}\right)\right) / \log 2,
$$

where $\operatorname{order}_{\mathrm{N}}$ denotes the $\mathrm{L}^{2}$-norm error at the final time $\mathrm{T}$ with the total number of elements equal to J.

Example 4.1. We first consider the viscous Burger's equation (1.1) with the initial condition

$$
u(x, 0)=2 v \frac{\pi \sin (\pi x)}{\sigma+\cos (\pi x)} .
$$

The initial condition for equation $(2.4) w(x, 0)$ can be derived and expressed explicitly as

$$
w(x, 0)=\sigma+\cos (\pi x) .
$$

The exact solution of this problem is obtained as

$$
u(x, t)=\frac{2 v \pi e^{-p i^{2} v t} \sin (\pi x)}{\sigma+e^{-p i^{2} v t} \cos (\pi x)}, x \in(0,1) .
$$

The number of the elements $J$ is defined as $J=2^{m}$ with $m=3,4,5,6$. We choose the time step as $\Delta \mathrm{t}=10^{-3}$. Tables 1-3 list the $\mathrm{L}^{2}$ errors and their numerical orders with different values of $v$, where $v=0.1,0.01$, and 0.001 , respectively. In each table the parameter $\theta$ is taken as $\theta=0.2,0.5$, and 1.0. From these tables, we can observe the different convergence with different $\theta$. For $\theta \neq \frac{1}{2}$, the suboptimal accuracy of $\left(k+\frac{1}{2}\right)$-th order for odd $k$ and optimal accuracy of $(k+1)$-th order with for even $k$ are obtained. For $\theta=\frac{1}{2}$, the suboptimal accuracy of $k$-th order for odd $k$ and optimal $(k+1)$-th order with for even $k$ can be obtained. This coincides with Theorem 2.4 and shows the sharpness of theoretical results. 
Example 4.2. The second test problem is as the same as the Example 4.1 but with different initial condition

$$
u(x, 0)=\sin (\pi x), \quad 0 \leqslant x \leqslant 1 .
$$

The exact solution of this equation is in the form of the infinite series

$$
u(x, t)=4 \pi v \frac{C}{D+E}
$$

where $C=\sum_{n=1}^{\infty} n I_{n}\left(\frac{1}{2 \pi v}\right) \sin (n \pi x) \exp \left(-n^{2} \pi^{2} v t\right), D=2 \sum_{n=1}^{\infty} I_{n}\left(\frac{1}{2 \pi v}\right) \cos (n \pi x) \exp \left(-n^{2} \pi^{2} v t\right), E=I_{0}\left(\frac{1}{2 \pi v}\right)$, and $I_{n}(x)$ is the first type of the $n$-th modified Bessel function. Here $n=50$ is used as an approximation to the infinite sum. With the Hopf-Cole transformation, the initial condition for heat equation (2.2) can be obtained as

$$
w(x, 0)=\exp \left\{-\frac{1-\cos (\pi x)}{2 \pi v}\right\}
$$

In order to compare the proposed LDG method with the exact solution and several existing numerical schemes, which are fully implicit finite difference method (IFDM) [4], a mixed finite difference, and boundary element method (BEM) [5]. The LDG scheme is chosen as $\mathrm{P}^{2}$ element with $\theta=0.5$. We divide the domain into $\mathrm{J}=25$ elements and take the time step as Example 4.1. The finial computation time is taken as $T=2$ with different viscosity $v=0.1$ and 0.01 . We list the numerical results and exact solutions at $x=0.1,0.3,0.5$, and 0.9 in Table 4 . We would like to notice that the obtained results match well with the exact solutions. And the numerical solutions by the LDG method generalized alternating fluxes are much more accurate than other numerical solutions.

Finally we plot the numerical solutions for $v=0.1$, and 0.01 at different times $T=0.2,0.5,1.0$, and 2.0 in Fig. 1. The plots show that the numerical solution exhibits the correct physical characteristic of the problem. Our algorithm has the ability to capture the shock for modest values of kinematic viscosity, $v=0.01$. In order to show the physical behavior of the given problem, we also give surface plots of the computed solutions for different $v$ in Fig. 2.

\section{Summary}

In this paper, we have applied the LDG method with generalized alternating flux for solving the Burger's equation. The nonlinear Burger's equation is transformed into linear heat equation by using Hopf-Cole transformation. The linear diffusion equation is then discretized by LDG method which results in a stiff system of first order ordinary differential equations. This system of ordinary differential equations is solved by exponential matrix method analytically. Numerical results are compared with exact solutions at different times. The obtained results confirm that our LDG method is a powerful and reliable method for capturing the shock phenomenon. The methods extended to higher dimensional case will be our future work.

\section{Acknowledgment}

R. Zhang's works was partially supported by the National Nature Science Foundation of China (Grant No. 61703290), the Key Laboratory Fund of National Defense Science and Technology (Grant Nos. 6142A050202), and China Scholarship Council. X. Yu's work was supported by National Natural Science Foundation of China (Grant No. 11571002), the Science Foundation of China Academy of Engineering Physics (Grant No. 2013A0202011, 2015B0101021) and the Defense Industrial Technology Development Program (Grant No. B1520133015). Z. Wang's work was supported by the National Natural Science Foundation of China (Grant No. 61573008). 


\section{References}

[1] G. Arora, V. Joshi, A computational approach using modified trigonometric cubic B-spline for numerical solution of Burgers' equation in one and two dimensions, Alex. Eng. J., 57 (2017), 1087-1098. 1

[2] G. Arora, B. K. Singh, Numerical solution of Burgers' equation with modified cubic B-spline differential quadrature method, Appl. Math. Comput., 224 (2013), 166-177. 1

[3] E. Ashpazzadeh, B. Han, M. Lakestani, Biorthogonal multiwavelets on the interval for numerical solutions of Burgers' equation, J. Comput. Appl. Math., 317 (2017), 510-534. 1

[4] A. R. Bahadir, Numerical solution for one-dimensional Burgers' equation using a fully implicit finite-difference method, Int. J. Appl. Math., 1 (1999), 897-909. 1, 4.2

[5] A. R. Bahadir, M. Sağlam, A mixed finite difference and boundary element approach to one-dimensional Burgers' equation, Appl. Math. Comput., 160 (2005), 663-673. 1, 4.2

[6] F. Bassi, S. Rebay, A High-Order Accurate Discontinuous Finite Element Method for the Numerical Solution of the Compressible Navier-Stokes Equations, J. Comput. Phys., 131 (1997), 267-279. 1

[7] J. Caldwell, P. Wanless, A. E. Cook, A finite element approach to Burgers' equation, Appl. Math. Modelling, 5 (1981), 189-193. 1

[8] S. Q. Chen, Y.-T. Zhang, Krylov implicit integration factor methods for spatial discretization on high dimensional unstructured meshes: Application to discontinuous Galerkin methods, J. Comput. Phys., 230 (2011), 4336-4352. 3.1

[9] Y. Cheng, X. Meng, Q. Zhang, Application of generalized Gauss?Radau projections for the local discontinuous Galerkin method for linear convection-diffusion equations, Math. Comp., 86 (2017), 1233-1267. 1, 2.2

[10] Y. Cheng, Q. Zhang, Local Analysis of the Local Discontinuous Galerkin Method with Generalized Alternating Numerical Flux for One-Dimensional Singularly Perturbed Problem, J. Sci. Comput., 72 (2017), 792-819. 1

[11] B. Cockburn, C.-W. Shu, The Local Discontinuous Galerkin Method for Time-Dependent Convection-Diffusion Systems, SIAM J. Numer. Anal., 35 (1998), 2440-2463. 1

[12] J. D. Cole, On a quasi-linear parabolic equation occurring in aerodynamics, Quart. Appl. Math., 9 (1951), 225-236. 1

[13] Y. Gao, L.-H. Le, B.-C. Shi, Numerical solution of Burgers' equation by lattice Boltzmann method, Appl. Math. Comput., 219 (2013), 7685-7692. 1

[14] E. Hopf, The partial differential equation $u_{t}+u_{x}=v u_{x x}$, Comm. Pure Appl. Math., 3 (1950), 201-230. 1

[15] P. Huang, A. Abduwali, The Modified Local Crank-Nicolson method for one-and two-dimensional Burgers' equations, Comput. Math. Appl., 59 (2010), 2452-2463. 1

[16] R. Jiwari, A hybrid numerical scheme for the numerical solution of the Burgers' equation, Comput. Phys. Commun., 188 (2015), 59-67. 1

[17] R. Jiwari, R. C. Mittal, K. K. Sharma, A numerical scheme based on weighted average differential quadrature method for the numerical solution of Burgers' equation, Appl. Math. Comput., 219 (2013), 6680-6691. 1

[18] A. H. Khater, R. S. Temsah, M. M. Hassan, A Chebyshev spectral collocation method for solving Burgers'-type equations, J. Comput. Appl. Math., 222 (2008), 333-350. 1

[19] S. Kutluay, A. R. Bahadir, A. Özdeş, Numerical solution of one-dimensional Burgers equation: explicit and exact-explicit finite difference methods, J. Comput. Appl. Math., 103 (1999), 251-262. 1

[20] X. Meng, C.-W. Shu, B. Wu, Optimal error estimates for discontinuous Galerkin methods based on upwind-biased fluxes for linear hyperbolic equations, Math. Comp., 85 (2016), 1225-1261. 1

[21] V. Mukundan, A. Awasthi, A Higher Order Numerical Implicit Method for Non-Linear Burgers' Equation, Differ. Equ. Dyn. Syst., 25 (2017), 169-186. 1

[22] T. Öziş, E. N. Aksan, A. Özdeş, A finite element approach for solution of Burgers' equation, Appl. Math. Comput., 139 (2003), 417-428. 1

[23] M. Seydaoğlu, U. Erdoğan, T. öziş, Numerical solution of Burgers' equation with high order splitting methods, J. Comput. Appl. Math., 291 (2016), 410-421. 1

[24] L. Shao, X. L. Feng, Y. N. He, The local discontinuous Galerkin finite element method for Burger's equation, Math. Comput. Modelling, 54 (2011), 2943-2954. 1

[25] M. Tamsir, V. K. Srivastava, R. Jiwari, An algorithm based on exponential modified cubic B-spline differential quadrature method for nonlinear Burgers' equation, Appl. Math. Comput., 290 (2016), 111-124. 1

[26] H. T. Xie, J. W. Zhou, Z. Jiang, X. Y. Guo, Approximations for Burgers equations with C-N scheme and RBF collocation methods, J. Nonlinear Sci. Appl., 9 (2016), 3727-3734. 1

[27] R. P. Zhang, X. J. Yu, G. Z. Zhao, The local discontinuous Galerkin method for Burger's-Huxley and Burger's-Fisher equations, Appl. Math. Comput., 218 (2012), 8773-8778. 1

[28] R. P. Zhang, X. J. Yu, G. Z. Zhao, Modified Burgers' equation by the local discontinuous Galerkin method, Chinese Phys. B, 22 (2013), 106-110. 1 